\title{
Shape Classification for Micro and Nanostructures by Image Analysis
}

\author{
F. Robert-Inacio, G. Delafosse and L. Patrone \\ Additional information is available at the end of the chapter
}

http://dx.doi.org/10.5772/50226

\section{Introduction}

In many fields considering information at microscale or nanoscale requires to achieve an automatic shape classification by image analysis, because of the amount of particles, on the one hand, and because of their size and reachability. This shape classification can be set up in $2 \mathrm{D}$ or in $3 \mathrm{D}$. In 2D, images are generally provided by a scanning electron microscope (SEM). In $3 \mathrm{D}$, different means can be used, such as reconstruction from a set of $2 \mathrm{D}$ sections or as direct tridimensionnal acquisition, for example by an atomic force microscope (AFM). Depending on the microscope resolution, shape studies can be lead either at nanoscale or at microscale. In this chapter, several shape parameters are defined and examples are given in two different fields of application: nanoelectronics and nuclear power. Both applications are achieved in 2D and 3D.

\section{Shape classification: state of the art}

In pattern recognition, an important field of interest concerns shape classification. Most of the methods used to reach this aim, consist in evaluating some geometrical features of the shapes under study, in order to determine which kind of shape family they belong to. For example, symmetry according to a point [23][24][47][48] can be measured by several means, such as Minkowski's, Besicovitch's, Winternitz's or Blaschke's coefficients [5][19]. Other applications require the evaluation of the symmetry degree according to an axis [31][42][43]. These studies are realized in 2D, 3D or arbitrary dimensions. And finally, circularity, elongation and so on [21][26], can be estimated in order to classify shapes. Classification methods can be shared in two different sets: the first one processing on natural images in gray levels or in color, in $2 \mathrm{D}$ or $3 \mathrm{D}$, and the second one on binary images describing well-defined objects. In the last case, natural images need to be pre-processed in order to identify shapes to study. Our method is one of this kind, and we are going to study the similarity between $2 \mathrm{D}$ or $3 \mathrm{D}$ objects. 


\section{Shape parameters in $2 \mathrm{D}$ and $3 \mathrm{D}$}

Several means can be used to describe shapes: circularity parameter, fractal degree of a 2D shape, polar study of the 2D boundary, peak dispersion on a surface in $3 \mathrm{D}$ or study of height variations in $3 \mathrm{D}$.

\subsection{Circularity parameters}

Let us start these studies by considering a classical shape parameter enabling to estimate the circularity degree of an object in two dimensions. It is based on a simple observation about circular objects. For a disk $D$ of radius $R$, the perimeter $P(D)$ is given by:

$$
P(D)=2 \pi \cdot R
$$

and the surface area $S(D)$ :

$$
S(D)=\pi \cdot R^{2}
$$

It yields that the circularity parameter $C P$, defined, for any object $X$, by:

$$
C P(X)=\frac{P(X)^{2}}{4 \pi \cdot S(X)}
$$

is equal to 1 , if and only if $X$ is a disk.

\begin{tabular}{lccc}
\hline \hline Shape & Perimeter $P$ & Surface area $S$ & $C P$ \\
\hline \hline Square $X_{1}$ & $4 \cdot l$ & $l^{2}$ & $\frac{4}{\pi} \approx 1.273$ \\
Hexagon $X_{2}$ & $6 . l$ & $3 \cdot l^{2} \cdot \cos \frac{\pi}{6}$ & $\frac{3}{\pi \cdot \cos \frac{\pi}{6}} \approx 1.103$ \\
Hexagon $X_{2}(\alpha)$ with a triangular concavity & $(6+\alpha) \cdot l$ & $\left(3-\frac{\alpha^{2}}{2}\right) \cdot l^{2} \cdot \cos \frac{\pi}{6}$ & $\frac{(6+\alpha)^{2}}{2 \pi\left(6-\alpha^{2}\right) \cdot \cos \frac{\pi}{6}}$ \\
Rectangle $X_{3}$ & $2 . l \cdot(1+t)$ & $t \cdot l^{2}$ & $\frac{(1+t)^{2}}{\pi t}>\frac{4}{\pi}$ \\
Rectangle $X_{3}(\alpha)$ with a square concavity & $2 l \cdot(1+t)$ & $l^{2} \cdot\left(t-\alpha^{2}\right)$ & $\frac{(1+t)^{2}}{\pi\left(t-\alpha^{2}\right)}$ \\
Rectangle $X_{3}^{\prime}(\alpha)$ with a rectangular concavity & $P\left(X_{3}\right)+\alpha$ & $S\left(X_{3}\right)-\frac{\alpha(k-\alpha)}{4}$ & $\frac{\left[P\left(X_{3}\right)+\alpha\right]^{2}}{4 \pi \cdot S\left(X_{3}\right)-\pi \cdot \alpha(k-\alpha)}$ \\
\hline
\end{tabular}

Table 1. CP values for the set of shapes of Fig. 7

In this way, we can see that $C P$ increases when $X$ becomes less close to a disk in shape. Fig. 8 illustrates the results given in Table 1 . The shapes under study are:

- A square $X_{1}$ of edge length 1 ,

- A regular hexagon $X_{2}$ of edge length 1 ,

- A regular hexagon $X_{2}(\alpha)$ with a triangular concavity (Fig. 1)

- A rectangle $X_{3}$ of height length $l$, and width length $t . l, t>1$

- A rectangle $X_{3}(\alpha)$ with a square concavity (Fig. 2)

- A rectangle $X_{3}^{\prime}(\alpha)$ with a rectangular concavity (Fig. 3)

Let us notice that the following relation is true, whatever the connected shape $X$ :

$$
C P(X) \geq C P(\operatorname{ch}(X))
$$

where $\operatorname{ch}(X)$ denotes the convex hull of $X$. 

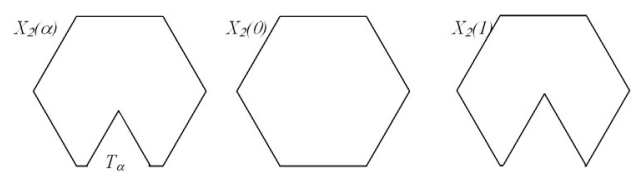

Figure 1. Shape family of non convex sets $X_{2}(\alpha)$ of convex hull defined by a regular hexagon, with a single triangular concavity $T_{\alpha}$ (equilateral triangle of edge length $\alpha . l$ with $\left.0 \leq \alpha \leq 1\right) \cdot X_{2}(0)$ and $X_{2}(1)$ are the two extreme shapes of the family

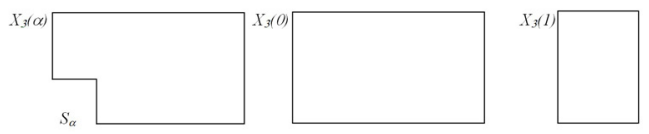

Figure 2. Shape family of non convex sets $X_{3}(\alpha)$ of convex hull defined by a rectangle, with a single square concavity $S_{\alpha}$ (square of edge length $\alpha . l$ with $\left.0 \leq \alpha \leq 1\right) . X_{3}(0)$ and $X_{3}(1)$ are the two extreme shapes of the family

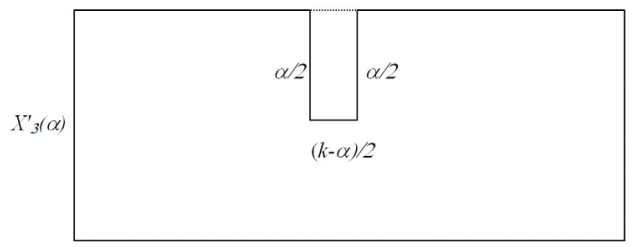

Figure 3. Definition of $X_{\alpha}$ according to a scalar value $\alpha, \alpha \in[0, k]$

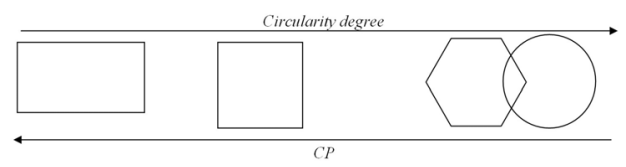

Figure 4. Classification of shapes $X_{1}, X_{2}$ and $X_{3}$ from the less to the most circular one, according to $C P$

Actually, $P(X) \geq P(\operatorname{ch}(X))$ and $S(X) \leq S(\operatorname{ch}(X))$. So we get the relation of eq. 4 .

Furthermore, the parameter $C P$ presents some interesting features such as invariance under affine transformations such as translations, scaling and rotations. It is then quite obvious that shape studies can be led without taking into account neither shape position and orientation, nor scale. As the parameter value is only related with the perimeter and the surface area, it can be interesting to pay more attention to the evolution of $C P$, when shapes are damaged by one or more concavities. We call concavity of $X$ a connected set of points included in $\operatorname{ch}(X)$ but not in $X$, having a non-empty intersection between its boundary and those of $\operatorname{ch}(X)$. In this way, holes are not allowed. Furthermore, we assume that two concavities of a same set $X$ have an empty intersection.

The concavity number is not really preponderant as it is always possible to find an equivalent concavity to a set of alterations, in terms of perimeter and surface area. In other words, the estimation of $C P$ is the same, when considering a single concavity $C$ or a set of concavities $C_{i}$, as far as the two following assumptions are satisfied: 


$$
P(C)=\sum_{i=1}^{N} P\left(C_{i}\right)
$$

and

$$
P(B d(\operatorname{ch}(X)) \cap B d(C))=P\left(B d(\operatorname{ch}(X)) \cap\left(\bigcup_{i=1}^{N} C_{i}\right)\right)
$$

where $B d(X)$ is the boundary of $X, S(C)=\sum_{i=1}^{N} S\left(C_{i}\right)$.

In this way, if $X$ is a convex shape:

$$
C P(X \backslash C)=C P\left(X \backslash \bigcup_{i=1}^{N} C_{i}\right)
$$

A second circularity parameter can be defined by considering the ratio between radii of the
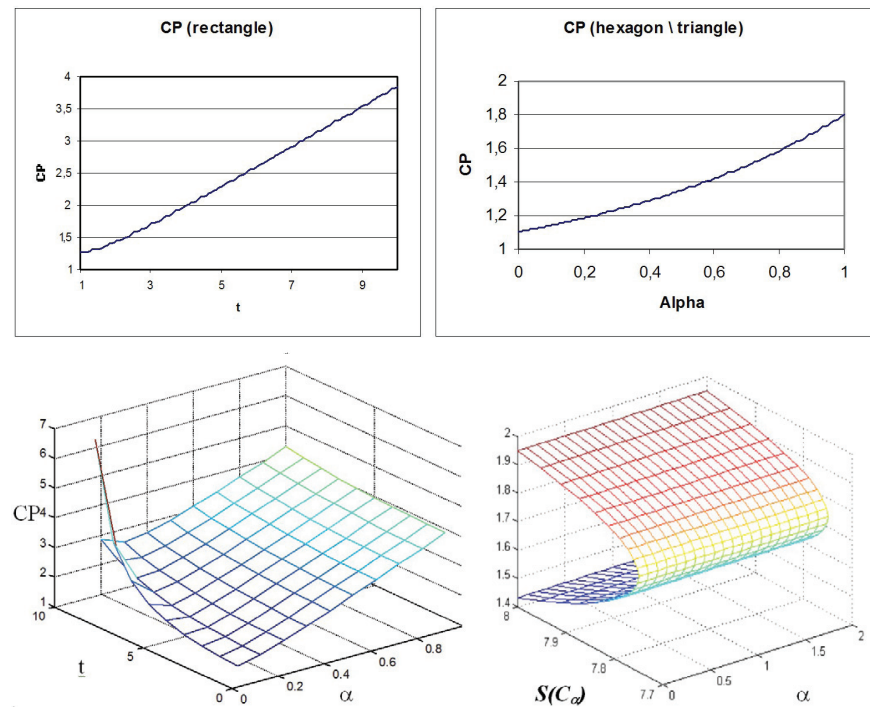

Figure 5. a) Evolution of $C P$ values according to the elongation $t$ of rectangular shapes, b) Evolution of $C P$ according to $\alpha$ for a regular hexagon with a triangular concavity, c) Evolution of $C P$ according to $\alpha$ and $t$ for a rectangle with a square concavity, d) Evolution of $C P$ according to $\alpha$ and $S\left(C_{\alpha}\right)$ for a rectangle with a square concavity

inscribed and circumscribed disks to the shape under study. If $r(X)$ and $R(X)$ are respectively the inscribed and circumscribed disks radii, then $C P 2(X)$ is defined as follows:

$$
C P 2(X)=\frac{r(X)}{R(X)}
$$

Fig. 6 illustrates the inscribed disk and circumscribed disk positions for a shape. While the circumscribed disk is unique, the inscribed disk can be located at different positions. 

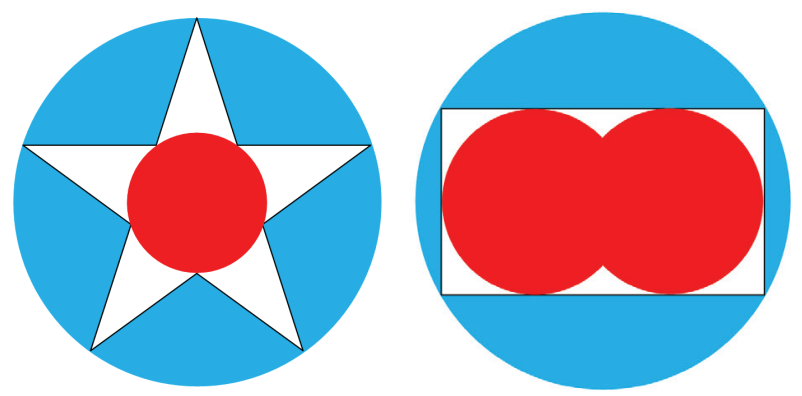

Figure 6. Disks associated to a shape (star or rectangle): in blue, circumscribed disk, in red, inscribed disk

$C P 2$ values belong to $[0,1]$ as they are positive values and $C P 2(X)$ is equal to 1 if $X$ is a disk. In this case, the circumscribed and the inscribed disks are equal to $X$, that gives the maximal value for $C P 2$.

The inscribed disk can be easily defined by using a euclidean distance map [16] that gives for each point of $X$ its distance to the boundary. The maximal values of such a map are located at inscribed disk centers. The circumscribed disk can be determined by using the circumscribed disk algorithm (see section 3.2.2).

\subsection{Shape classification according to a given shape}

In order to achieve shape classification according to a reference shape, a similarity parameter $P$ is defined for any pair of convex shapes $(X, Y)$ by considering two scale ratios. The first one gives the smallest homothetic set of $X$ containing $Y$, and the second one, the smallest homothetic set of $Y$ containing $X$. Thus, let us define the following function $S_{X}(Y)$ :

$$
\begin{aligned}
S_{X}: & \mathbb{K} \longrightarrow \mathbb{R}^{+} \\
& Y \mapsto S_{X}(Y)
\end{aligned}
$$

where

$$
S_{X}(Y)=\inf \left\{k>0 ; Y \subset_{t} k \cdot X\right\}
$$

$\subset_{t}$ means "included in, regardless to any translation". And then, a definition of the similarity parameter can be:

$$
P(X, Y)=\frac{S_{Y}(X)}{S_{X}(Y)} \cdot \frac{\mu(X)}{\mu(Y)}
$$

where $\mu$ is the surface area measure. The parameter properties are the following:

1. if $X \subset_{t} Y$ then $P(X, Y)$ belongs to $\left.] 0,1\right]$

2. $P$ is invariant by translation

3. $P$ is invariant by scaling

4. if $X$ and $Y$ are of the same shape regardless to a positive scale ratio then $P(X, Y)=P(Y, X)=1$

5. $P(X, Y)=P(Y, X)^{-1}$ 


\begin{tabular}{cccccc}
\hline \hline Shape $X$ & $X_{i}$ & $S_{X}(Y)$ & $S_{Y}(X)$ & $\mu(X)$ & $P_{Y}(X)$ \\
\hline \hline Square & $X_{1}$ & 0.84375 & 2.09677 & 961 & 0.18736 \\
Pentagon & $X_{2}$ & 0.81250 & 1.45455 & 829 & 0.22436 \\
Parallelogram & $X_{3}$ & 0.93750 & 2.40741 & 891 & 0.16811 \\
Rectangle 1 & $X_{4}$ & 0.96875 & 1.69767 & 1118 & 0.30909 \\
Rectangle 2 & $X_{5}$ & 0.78125 & 2.56000 & 825 & 0.12198 \\
Triangle & $X_{6}$ & 0.71875 & 2.41026 & 585 & 0.08452 \\
Ellipse 1 & $X_{7}$ & 0.68750 & 2.17500 & 691 & 0.10585 \\
Ellipse 2 & $X_{8}$ & 0.84375 & 4.33333 & 365 & 0.03445 \\
Circle & $X_{9}$ & 0.96875 & 1.58537 & 1320 & 0.39087 \\
Regular hexagon & $X_{10}$ & 0.93750 & 1.39130 & 1320 & 0.43094 \\
\hline
\end{tabular}

Table 2. Results of computation for $P_{Y}(X), \mu(Y)=2064$

In order to set up a process of shape classification, the two most interesting properties of the similarity parameter $P$ are given in (1) and (4). In other words, under the assumption that $X \subset_{t} Y$, we can compute a similarity parameter $P_{Y}$, estimating for each convex set $X$, its similarity degree to $Y$. By this way, the values reached by $P_{Y}$ belong to the interval $\left.] 0,1\right]$. So we are able to arrange in order the sets $X$ under study, from the less similar to $Y$ to the most similar. Let us study the following example. The reference shape $Y$ that has to be compared with the others is a hexagon. Then, let us consider ten arbitrary shapes $X_{i}$, such as a square, a pentagon, a parallelogram, two rectangles, a triangle, two ellipses, a circle and a regular hexagon. We compute the parameter $P_{Y}$ for each of these ten arbitrary shapes, and, by this way, we are able to define a partial order for this set of shapes, according to their similarity degree to the hexagon $Y$. Fig. 7a describes the convex sets $Y$ and $X_{i}$ and Table 2 gives the computational results. In this way, the previous results can be illustrated as shown in Fig. $7 \mathrm{~b}$.

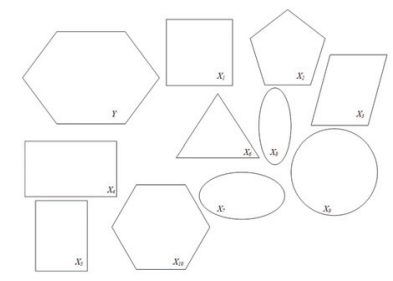

(a) Convex sets under study: hexagon $Y$ and arbitrary shapes $X_{i}$

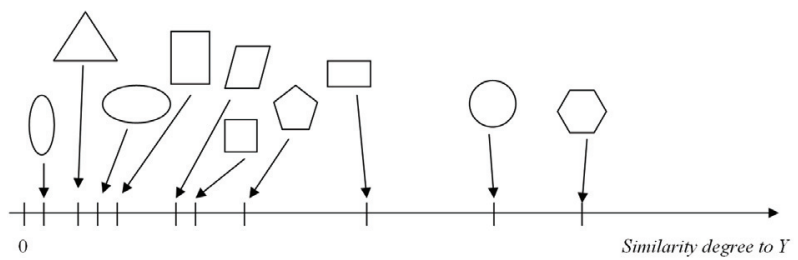

(b) Convex sets under study: hexagon $Y$ and arbitrary shapes $X_{i}$

Figure 7. Shape study on a set of simple sets

About this classification, we can note that the closest set in shape to the hexagon $Y$ is the regular hexagon. Furthermore, the second one is the circle, which is close to every regular polygon. And finally, the third one is the rectangle oriented in the horizontal direction, like the hexagon $Y$. On the opposite side, the less close shape to $Y$ is an ellipse oriented in the vertical direction. Thus, the similarity parameter seems to take into account some geometrical features such as the orientation and the elongation. Then, if the reference shape is a disk $P$ estimates the circularity degree. Furthermore this parameter can be used in 2D or in 3D. It is enough to change the area measure $\mu$ into a volume measure to obtain the tridimensionnal version of the parameter $P$. 


\subsubsection{Implementation of the similarity parameter}

In order to implement the similarity parameter, we must set up fast and easy algorithms determining the features of circumscribed convex shapes. In this way, the scale ratios required for the similarity degree estimation will be easily evaluated. Assuming that we have at our disposal an algorithm called CircumRatio $(X, Y)$, determining the scale ratio to apply to a convex set $X$ to circumscribe it to a convex $\operatorname{set} Y$, the algorithm estimating the similarity degree between two shapes is the following:

Algorithm

$k=\operatorname{CircumRatio}(X, Y)$

$k^{\prime}=\operatorname{CircumRatio}(Y, X)$

Compute the two surface areas of $X$ and $Y$

Compute the similarity degree of $X$ and $Y$

First of all, let us consider the circumscribed disk algorithm that allows the user to compute and design the minimal disk containing a given planar object $X$. The extension of this algorithm to convex sets is the algorithm used to evaluate the scale ratios, and then, the similarity parameter.

\subsubsection{The circumscribed disk algorithm}

The main results concerning the circumscribed disk algorithm are:

\section{Proposition 1}

$X$ is a compact set of $\mathbb{R}^{2}$ and $B(x, R]$, the closed ball whose center is $x$ and radius $R$. The following assertions are equivalent:

$$
\begin{gathered}
X \subset B(x, R] \\
x \in \bigcap_{y \in X} B(y, R] \\
x \in \bigcap_{y \in \partial X} B(y, R] \\
x \in \bigcap_{y \in E(X)} B(y, R]
\end{gathered}
$$

where $E(X)$ is the extreme point set of the convex hull of $X$. Let us recall that a point $a$ of a convex body $K$ is extreme if $\forall x, y \in K, a=\frac{x+y}{2} \Rightarrow x=y$. Fig. 8 is an illustration of the four previous assertions.

Then, the features of the circumscribed disk to any compact set are given by the following formulae:

$$
\begin{array}{r}
R(X)=\inf \{R>0, B(O, R] \ominus \partial X \neq \varnothing\} \\
a_{X}=\{a(X)\}=B(O, R(X)] \ominus \partial X
\end{array}
$$

$R(X)$ is the radius of the circumscribed disk and $a(X)$ its center. 


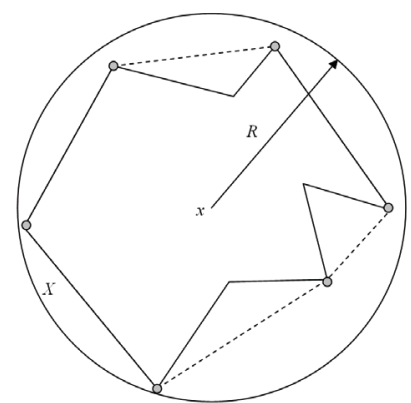

Figure 8. Illustration of Proposition 1: in plain line, the compact set $X$, in dashed line, its convex hull $C(X)$ and in gray, its extreme points $E(X)$

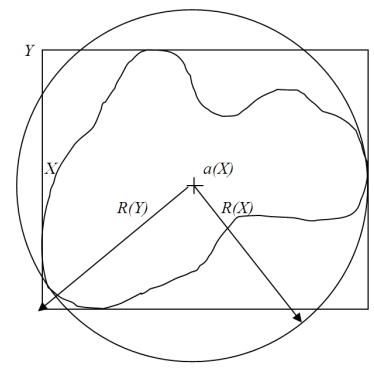

Figure 9. $R(X) \leq R(Y)$

Furthermore, let $R \geq R(X), a(X)$ is the ultimate eroded set of $B(O, R] \ominus \partial X$ by a closed ball of elementary radius $\epsilon>0$. Let us recall that the ultimate eroded set of a set $X$ by a set $Y$, is the last non-empty eroded set of $X$ in succesive erosions by $Y$. In other words, there exists a positive integer $n$ such that $X \ominus n Y \neq \varnothing$ and $X \ominus(n+1) Y=\varnothing$. In this way, $X \ominus n Y \neq \varnothing$ is the ultimate eroded set of $X$ by $Y$. From the previous properties, an algorithm can be described by the following steps:

\section{Algorithm 1}

1) Acquire a compact set $X$

2) Search $C(X)$, convex hull of $X$

3) Search $E(X)$, extreme point set of $C(X)$

4) For each point $x$ of $E(X)$

4-1) Draw the circle whose center is $x$ and radius $R$

4-2) Fill this circle with respect to the intersection set found yet

4-3) Delete all points which do not belong to the new intersection

5) Ultimate eroded set of the disk intersection

Some classical algorithms exist for the convex hull computation [2][4]. For the determination of extreme points of this convex hull, we can consider that the convex hull is a polygon when working in a discrete space. Then, it is sufficient to determine for each vertex of this polygon if it is extreme or not, in other words, if it is convex or not. 
The computation of the ultimate eroded set enables not only to find the location of the center but also to evaluate the radius value. In fact, if $B\left(O, R_{0}\right]$ is the closed ball used to obtain the ultimate eroded set of $B(O, R] \ominus \partial X$, then the radius $R(X)$ is given by:

$$
R(X)=R-R_{0}
$$

The initial value $R$ can be chosen as half the length of the diagonal line of a circumscribed rectangle $Y$ to the given compact $X$. This value is actually the radius value $R(Y)$ of the circumscribed disk to the rectangle $Y$. As the rectangle $Y$ contains the compact set $X, X$ is also included in its circumscribed disk and $R(Y)$ is greater or equal to $R(X)$ (Fig. 9).

We can remark that solving the difficult problem of computing the circumscribed disk to any object is reduced to obtain the inscribed disk in a convex set. Actually, the convex set is $B(O, R] \ominus \partial X$ (that is convex because it is the intersection of convex sets) and, when computing the ultimate eroded set by $B\left(O, R_{0}\right]$, the resulting point is the center of the inscribed disk into $B(O, R] \ominus \partial X$ (the radius of this disk is $\left.R_{0}\right)$.

\subsubsection{Extension to the third dimension}

The similarity parameter can easily be extended to the third dimension by replacing the surface area measure by the volume measure of $3 \mathrm{D}$ objects. The definition is the following:

$$
P(A, B)=\frac{S_{A}(B)}{S_{B}(A)} \cdot \frac{V(A)}{V(B)}
$$

where $V$ is the volume measure. The circumscribed disk algorithm is based on theoretical results that are also true in $3 \mathrm{D}$. That is why the process can be set up in 3D.

\subsection{Circularity parameter and fractal degree}

The definition of $C P$ and the previous considerations on some particular shapes leads us to the conclusion that the circularity parameter $C P$ is able to estimate a ratio giving indications about the fractal degree [44] on shapes in 2D. It is indeed based on the computation of a ratio between the square of the perimeter and the surface area of the shape under study. In this case, $C P$ increases according to the perimeter while the surface area remains constant. This feature is appropriate to deduce information about the fractal degree for the shape under study, as fractal objects have the feature that their perimeters tend to an infinite value, whereas their surface areas are bounded. So the greater this parameter value, the more fractal the shape, in other words, shapes of a same surface area are more and more fractal while their perimeter increases. Fig. 10 shows that $C P$ increases in an exponential way, when the perimeter value grows. The initial value is computed for a disk of radius of 5 . Then the perimeter is approximately equal to 31.4159 and the surface area to 78.5398 , and obviously, $C P$ is equal to 1 .

As $C P$ depends on the square of the perimeter, its values tend to $+\infty$ when the perimeter increases.

$$
C P(X)=f\left(P(X)^{2}\right) \Rightarrow \lim _{P(X) \rightarrow+\infty} C P(X)=+\infty
$$




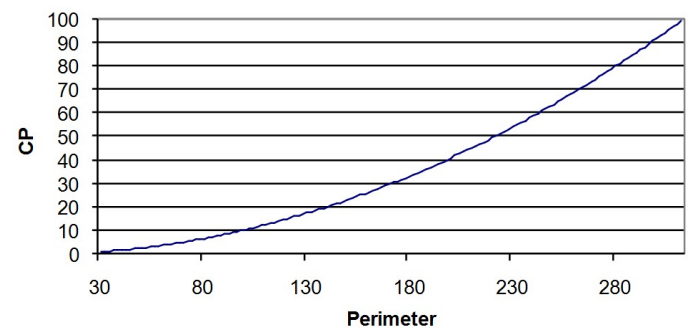

Figure 10. Evolution of $C P$ according to the perimeter

That induces that $C P$ estimates the fractal degree of a shape $X$. In other words, the higher the $C P$ value, the more fractal the shape $X$.

\subsection{Polar study of the boundary of $2 \mathrm{D}$ objects and estimation of the circularity degree}

This section presents a local estimation of the circularity degree for objects in 2D [37]. The method consists in drawing the boundary of the shape under study, in polar coordinates, according to an appropriate centre. This centre is chosen as the best-centred point amongst the set of centres of the inscribed disks in the shape. In other words, this centre is the centre of an inscribed disk and it is the closest one to the centroid of the shape under study. We will call this particular point the polar centre of the shape. In the three following examples, this polar centre coincides with the centroid.

The polar graphs corresponding to a square, a rectangle and a regular hexagon (Fig. 11) show a visual estimation of the circularity degree of those three shapes, whereas the standard deviation measures the dispersion of radius values around the average radius. The higher the standard deviation, the less circular the shape. Table 3 gives numerical results of average and standard deviation computation for the three shapes under study. Obviously, if the shape is a disk, the average value is equal to its radius and the standard deviation to 0 .

\begin{tabular}{lccc}
\hline \hline Shape & \multicolumn{3}{c}{ Square } \\
\hline \hline Average radius value & 5.7381 & 4.2000 & 4.5611 \\
Standard deviation of radius values & 0.6311 & 1.0596 & 0.2031 \\
Maximal distance to the average value & 1.3330 & 1.7000 & 0.4389 \\
\hline
\end{tabular}

Table 3. Visual estimation of the circularity degree from polar graphs

>From Table 3, shapes can be ordered from the less circular to the most circular, according to the standard deviation values: rectangle, square and regular hexagon. This result coincides with those obtained from the circularity parameter $C P$.

Fig. 11 shows that the circularity degree of a shape is closely linked to the amplitude of its polar graph. In other words, the maximal distance measured to the average value of each graph is another way to estimate the circularity degree, that is equivalent to the standard deviation evaluation, as shown in Table 3. 


\section{Superposition of polar graphs}

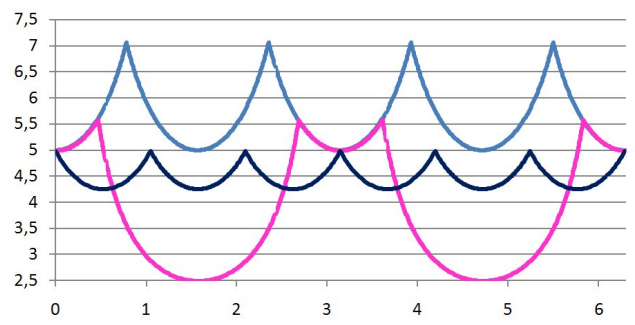

Figure 11. Boundary in polar coordinates of the three previous shapes (coarse angle sampling step). In dark blue, the hexagon, in light blue, the square and in pink, the rectangle

\subsection{Study of peak dispersion on a surface in 3D}

A surface can present several particular spots located at local maxima in terms of height. These spots are called peaks and they are characterised by their summit (single point) that is higher than its neighbours. Detection of peaks is achieved by considering a square sliding window of 5 pixels width. In order to study the peak dispersion on a given surface, the first step of the process consists of the computation of an Euclidean distance map [16], giving at each point of the surface its distance to the boundary. This distance map is computed on a projection of the surface in the plane $(O, x, y)$, defined in the introduction section as the plane of the corresponding grey-level image. Fig. 12 shows such a bounded surface whose projection is a square of 80 pixel-width and Fig. 13 is the associated Euclidean distance map. On this map, every point belonging to the surface outside is set to 0 and points of the surface are set to their Euclidean distance from the boundary. Then the surface is scanned in order to detect peaks as local maxima. Such a task is achieved by considering decreasing height values. Then each point is checked to determine if it is a local maximum or not. Three main peaks can be detected on this surface. Their features as well as those of the 2D centroid are given in Table 4 . The centroid is taken under consideration as it is a point representative of the whole shape in 2D. Furthermore, in this case, it is also the point where the distance map reaches its maximal value. In other words, this point can be considered as the centre of an inscribed disk into the 2D shape.
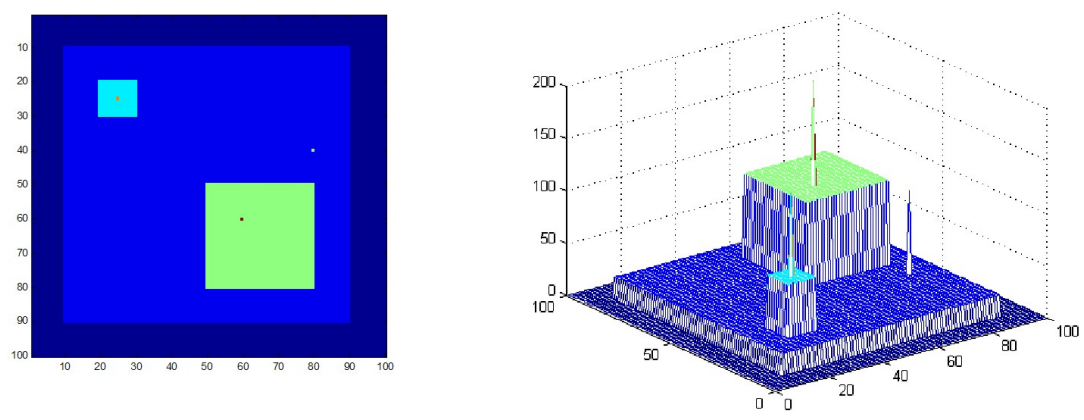

Figure 12. Overview of a bounded surface (left) and its 3D representation (right) 


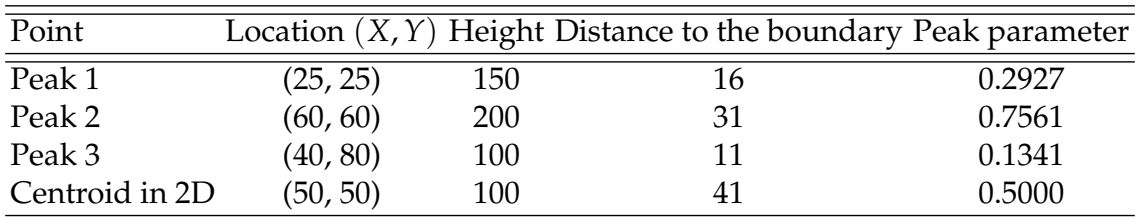

Table 4. Features of main peaks and centroid

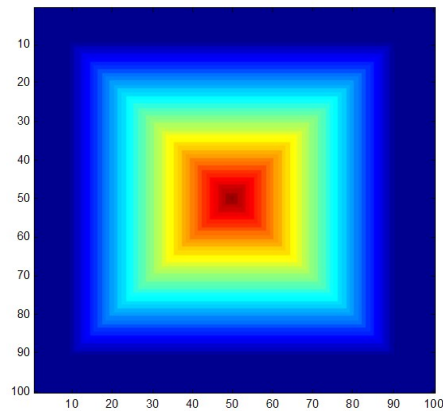

Figure 13. Associated Euclidean distance map

PP evolution according to peak height

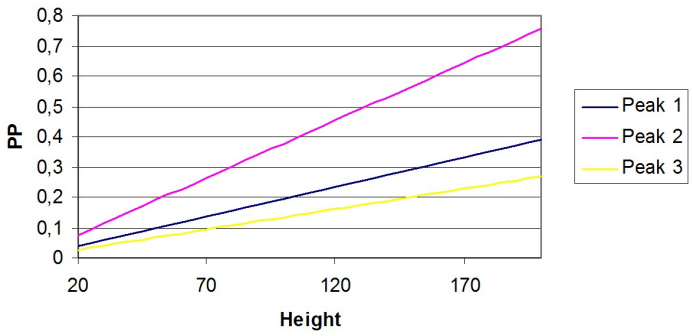

Figure 14. Influence of peak height on $P P$ values

$>$ From the previous features, a parameter can be associated to each peak. This peak parameter $P P$ is defined as follows:

$$
P P(p)=\frac{h(p) \cdot d(p)}{h \max \cdot d \max }
$$

for a peak $p$ of the shape $X$, with:

$$
h \max =\max \{h(m), m \in X\}
$$

and

$$
d \max =\max \{d(m), m \in X\}
$$

where $h(m)$ represents the height at the point $m$ and $d(m)$ the distance from the point $m$ to the boundary of $X$. In the previous case, $d \max =41$ and hmax $=200$. The parameter $P P$ is maximal (equal to 1 ) if the highest peak coincides with the point of maximal value on the Euclidean distance map. This parameter enables to estimate a combination between 
height and centring. Its values vary between 0 and 1 and a value close to 0 represents a non-significant peak in terms of height or centring, or both. The peak dispersion is then established by arranging in order all the significant peaks according to the value of parameter $P P$. In order to characterize the shape $X$, the parameter $\overline{P P}$ is determined as the average value of $P P$ on the peaks $p$ of $X$ :

$$
\overline{P P}(X)=\frac{1}{N} \sum_{p \in X} P P(p)
$$

where $N$ is the number of peaks $p$ of $X$. In the previous case:

$$
\overline{P P}(X)=0.3943
$$

\section{PP according to height and distance}

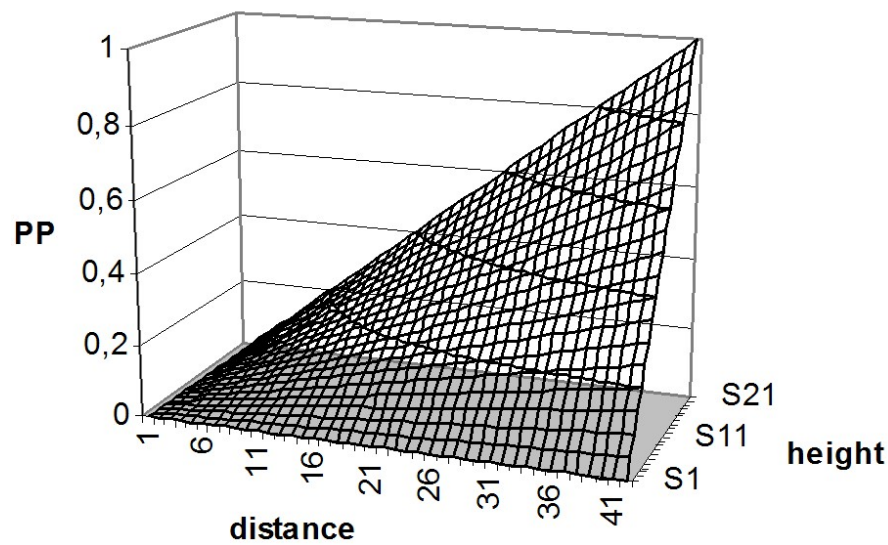

Figure 15. Influence of peak height and distance to the boundary on $P P$ values

The estimation of $P P$ at the maximal point of the distance map gives an evaluation of the centring degree of the shape in terms of height. Fig. 14 shows how the peak height influences the value of $P P$. The distance to the boundary is then preponderant to determine the coefficient of the slope. On Fig. 15, the influence of the distance and the height of a peak has been drawn for a maximal distance value dmax of 41 and a maximal peak height hmax of 200 (S1 corresponds to a height of $0, S 11$ to 100 and $S 21$ to 200), that are the characteristic values of the bounded surface $X$. This graph is representative of the surface and gives the features of centring and height of an arbitrary peak, whatever its position or height. It is then enough to read the corresponding value on the graph to determine $P P$.

Furthermore, a coefficient of centring CC can be estimated at each peak $p$ of $X$, described by the following formula:

$$
C C(p)=\frac{d(p)}{d \max }
$$

Table 5 gives the $C C$ values for the main peaks of $X$. 


\begin{tabular}{|c|c|}
\hline Peak $(X, Y)$ & $(25,25)(60,60)(40,80)$ \\
\hline Local estimation of height and centring combination & 1100 \\
\hline
\end{tabular}

Table 6. Height and centring combination $(H C C)$ at main peaks of $X$

a region of well-centred points at a medium height. The HCC values are more significant in this region than they are at peaks located at $(25,25)$ and $(40,80)$. For example, the centroid located at $(50,50)$ has a $H C C$ value equal to 4100 . But we must remember that the centroid is also the maximal point for the Euclidean distance map in this particular case.

Finally, the variation in height $\mathrm{VH}$ according to the distance to the edges can be estimated by computing the average value on a given level of the distance map. In other words, we consider all points at a given distance to the boundary and we compute the average height. This can be done for each distance value from 1 to the maximal value. Fig. 17 is the graph of $V H(X)$.

Unfortunately, such a graph (Fig. 17) makes the assumption that the bounded surface under study has an isotropic behaviour, as it is based on an average value computation that does not take into account orientation. It should be better to draw an equivalent surface in polar coordinates to unfold the original surface. The resulting information would show the variations of height according to the angle, with respect to the polar centre.

The peak dispersion on the considered bounded surface is then determined by setting up a process computing the heights (from the original grey-level image) and locations (from the Euclidean distance map) of the main peaks. That will define a simplified map of the surface, including only main information in terms of height.

\section{Application 1: sphericity of microscopic particles}

\subsection{Context}

V/HTR (Very/High Temperature Reactors) are advanced nuclear power reactors that employ spherical particles made of an uranium kernel surrounded by four different layers (Fig. 18) $[14,27,33]$. During fuel particle manufacturing, the coating process may generate non spherical particles and/or ceramic layers with abnormal thickness. For such particles, the fuel performances are highly decreased [12, 18]. That is why, it is necessary to set up a characterizing tool allowing, on the one hand, to estimate the thickness and sphericity degree of these fuel particles at each step of the fabrication and, on the other hand, to measure a statistically representative sample taken from fabrication batches containing millions of particles [13]. A classification of fuel particles has to be established in order to reject through statistical control HTR particles batches presenting too serious faults.

\subsection{Aim of the study}

HTR nuclear fuel particles are made of a kernel of uranium oxide of 500 ţm covered by a first layer of porous pyrocarbon (fission gas tank) and a layer of silicium carbide sandwiched between two layers of dense pyrocarbon. The aim of this study is to measure some geometrical features of such particles, in particular, the kernel diameter and sphericity, and the thickness 


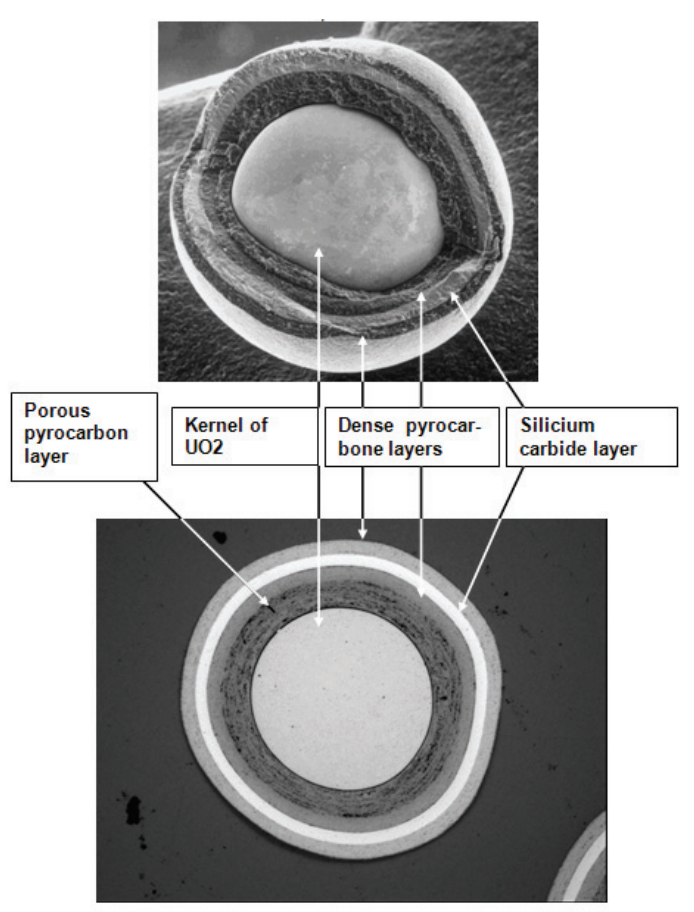

Figure 18. Fuel particle in $3 \mathrm{D}$ and polished cross-section

of each layer. The presented method is based on different thresholding processes enabling to extract the kernel or one of the layers from the image [37]. Then, an Euclidean distance map is computed on the binary images, giving for the one corresponding to the kernel its diameter, and for the others, an estimation of the layer thickness. An original shape parameter evaluating the sphericity degree of the kernel is then deduced from the distance map and its value is compared to the shape parameter computed from Féret's diameters. Furthermore, some information related to thickness is extracted from the layer distance maps. For example, the maximum, minimum and average thicknesses are computed from these maps, but a regular set of thickness values can be obtained according to angular sampling. This method enables an automatic control of the particle features in order to classify them in two sets : satisfactory particles and rejected particles [37].

The Euclidian distance mapping [16] is a method that estimates the minimal distance between each pixel of the background and the nearest object. It works on binary images (Fig. 19). The first distance map is computed inside the kernel in order to obtain for each of its points, its distance to the outside, and then, the centre of inscribed disk of the kernel. This centre is determined as the maximal value of the distance map, which represents the radius of the inscribed disk, and it is also considered as the real centre of the particle. After that, a second distance map is computed in order to calculate the kernel radius, by temporarily considering the centre of the particle as the only object of the image. Thus, this new distance map gives a set of concentric circles centred at the kernel centre (Fig. 20). The use of a border 

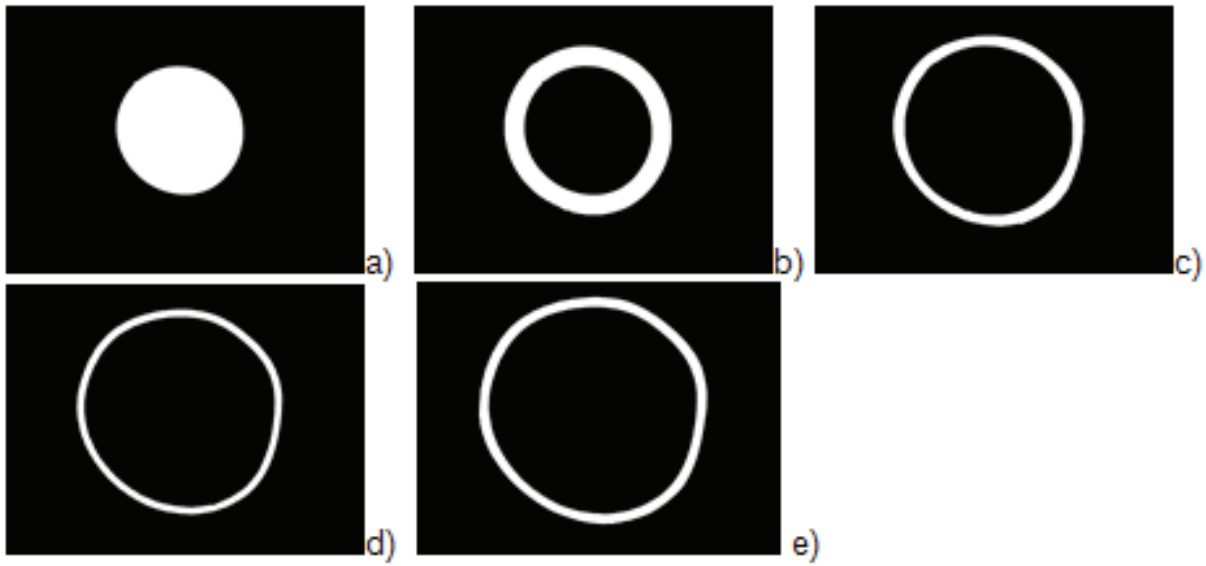

Figure 19. Binary images corresponding to a) the kernel, b) porous pyrocarbon, c) inner pyrocarbon, d) silicium carbide and e) outer pyrocarbon

follow-up method will reveal the kernel local radius by looking at the intersection between the binary image of the kernel and the last distance map. Finally, the process is extended to the surrounding layers by computing four other distance maps on the different binary images representing the different layers. The half thickness of a layer is then determined as the locally maximal values on the distance map. In this way, we obtain thickness measurements in every direction, that allow us to draw the polar graphs.
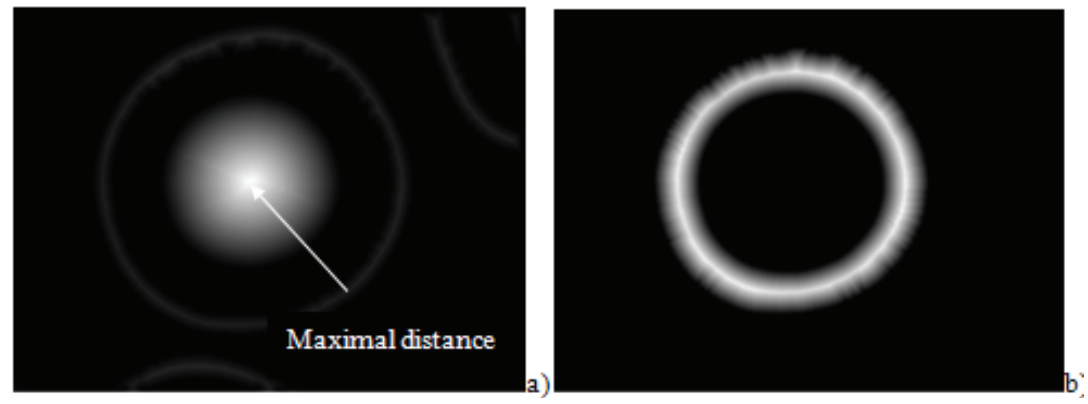

Figure 20. Distance maps associated with a) the kernel and b) the SiC layer

\subsection{Experimental results}

Fig. 21 shows polar graphs of the different layers and Fig. 22 presents the links between the particle and the polar graphs. Futhermore, Table 7 gives thickness results at the equatorial cross-section, in other words, theoretical and computed thickness results of each element of the particle. The minimum and maximum values for each element are useful in order to detect major local deformation of the particle, while the average value is more representative of the particle regularity. 

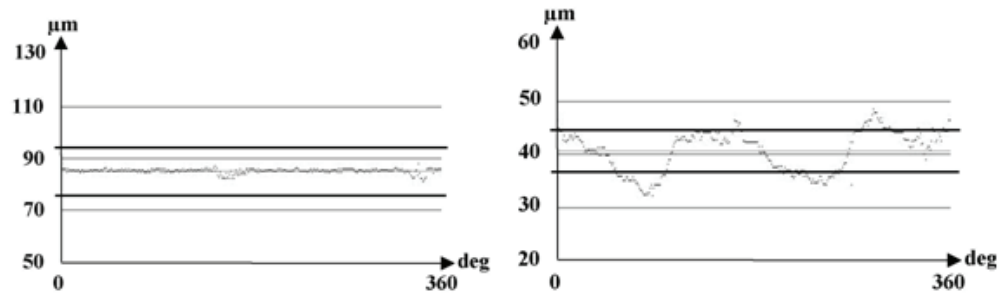

a

$\mathrm{b}$
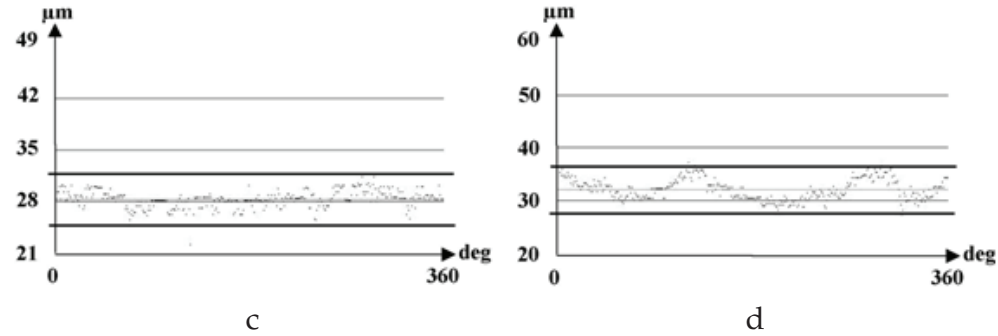

Figure 21. Real deposit of the different layers : a) Porous PyC, b) inner PyC, c) SiC and d) outer PyC

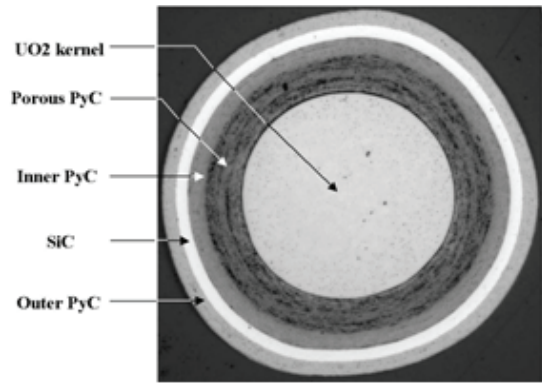

(a) Polished cross-section of a HTR fuel particle

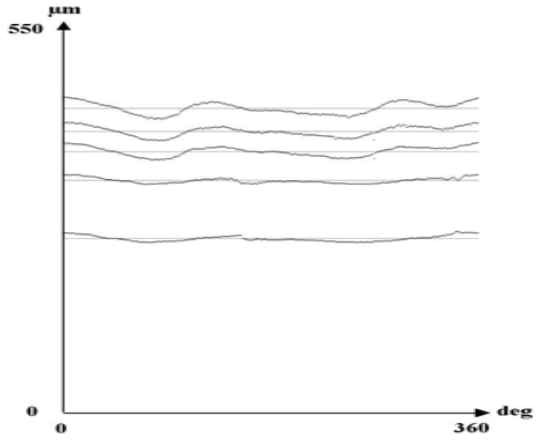

(b) Polar graph representing the kernel and the four layers boundaries of the particle

Figure 22. HTR fuel particle study

\begin{tabular}{ccccc}
\hline \hline Element & \multicolumn{4}{c}{ Thickness at the Equatorial Cross-section $(\mu \mathrm{m})$} \\
& Maximum & Minimum & Average & Theory \\
\hline \hline Kernel (radius) & 263.38 & 247.96 & 253.18 & $250 \pm 20$ \\
Porous PyC & 88.03 & 81.14 & 85.29 & $90 \pm 20$ \\
Inner PyC & 48.47 & 32.29 & 40.61 & $40 \pm 10$ \\
SiC & 31.44 & 20.21 & 28.40 & $35 \pm 7$ \\
Outer PyC & 37.20 & 27.22 & 32.12 & $40 \pm 10$ \\
\hline
\end{tabular}

Table 7. Thickness results appearing at the equatorial cross-section 
Table 8 gives the computed spherical results of a particle at different states of reconstruction. The results are given in percentages where $100 \%$ means that the particle is perfectly circular.

\begin{tabular}{ccc}
\hline \hline Element & \multicolumn{2}{c}{ Sphericity degree (\%) } \\
& CP & CP2 \\
\hline \hline Kernel (K) & 96.02 & 94.81 \\
$\mathrm{~K}+$ Porous PyC & 97.24 & 96.14 \\
$\mathrm{~K}+$ Inner PyC & 94.57 & 94.00 \\
$\mathrm{~K}+$ SiC & 94.27 & 94.25 \\
$\mathrm{~K}+$ Outer PyC & 94.06 & 93.80 \\
\hline
\end{tabular}

Table 8. Sphericity degree results at different levels of the particle

\section{Application 2: shape analysis on molecular islands of carbon chains in nanoelectronics}

\subsection{Self-assembly}

Self-assembly is an universal phenomenon, responsible for the structural organization of a system without external intervention. The molecular self-assembly is studied in order to obtain nanometer-sized structures in a bottom-up approach. This one consists in building a structure from individual base elements step by step.

Such an approach is conceivable for the realization of molecular layers since K. Blodgett and I. Langmuir have achieved the transfer of monolayers from water/air interface to solid surfaces[6, 7], thereby bequeathing their name to the method: the Langmuir-Blodgett (LB). Kuhn et al.[22] achieved in the 1970's nano-manipulation of molecules. To overcome the drawbacks of the LB method, J.Sagiv et al.[32] prepared SAMs by chemisorption, based on silane chemistry. With the same aim, Mallouk et al.[20] used crystalline chemistry. Since then, other techniques such as vapor deposition, nanolithography[34] have been developed for self-assembled monolayers (SAMs).

Except for the LB method, self-assembling molecules can be divided into three parts (fig. 23)[1].

Surface-active head group: This moiety will react with the surface. The choice of the head group depend on the surface used. Grafting can provide different chemical bonds (covalent, ionic, ...).

body group: Generally, the body is an alkyl, or derivatized-alkyl group. The ordering process is driven by van der Waals or electrostatic interaction.

Surface group: the choice of this group depends on the aim. This group gives its properties to the SAM (wettablility, reactivity, ...). 


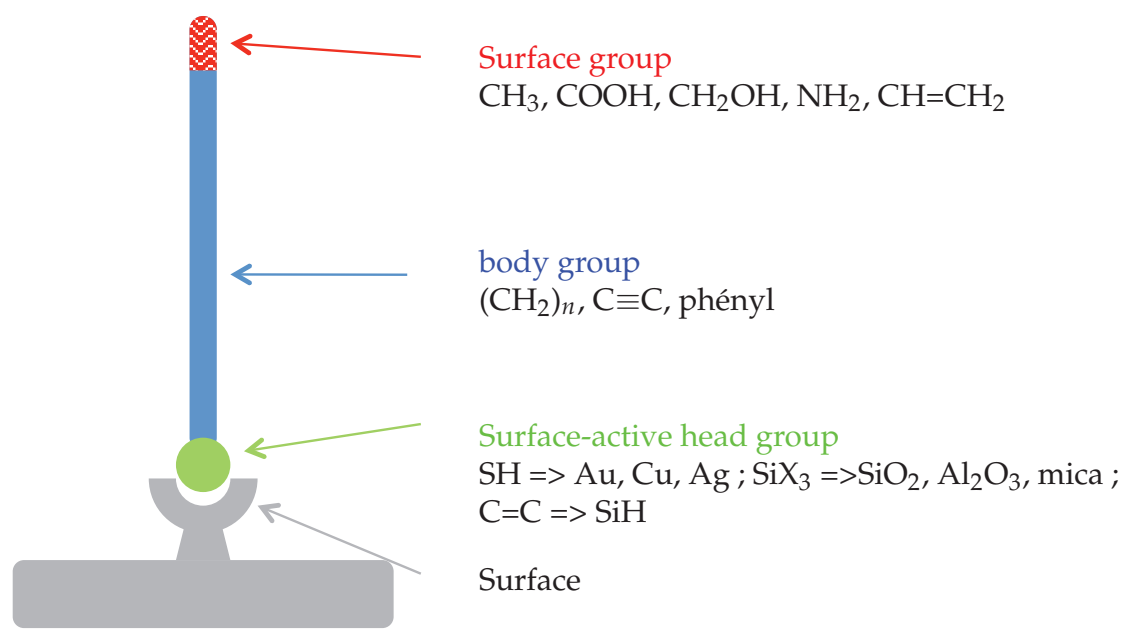

Figure 23. A schematic view of molecular structure [1]

Note that SAMs are more stable than LB films. Indeed, the latter are simply physisorbed, while self-assembled films, by their chemisorption, are more resistant to chemical attack and are more stable in temperature[45].

\subsubsection{Self-assembly: interest}

The interest of the self-assembly is in the control and the modification of the surface for specific applications. One can set the affinity of a surface with water or other solvents, such as protecting non-oxidized surfaces from water by making them hydrophobic[17]. SAMs also ensure biological compatibility for anchoring proteins[41] or conversely make bactericidal surface[38]. Note also the control of friction properties[25], the formation of very thin insulator[28,46]. The combined use of these techniques with others, such as photolithography, allows also the organization of carbon nanotubes on a surface[15]. Furthermore, sequential self-assembly of SAM can be used to create 3D structures[3, 29, 30, 45].

\subsubsection{Self-assembly: difficulties}

Although the exothermic reaction[1] $(\sim 1-2 \mathrm{eV})$ between the surface-active group and the substrate promotes the use of a maximum of docking sites, forming a compact and orderly SAM on a large scale is not easy. Indeed, to enable the interchain interactions, it is first necessary that the molecules are close enough, requiring a high density of grafted molecules. Alkyl chains are tilted with respect to the normal to the surface by an angle $(\alpha)$, that depends on the recovery rate and defects[39]. Generally the higher the coverage, the smaller is $\alpha$.

\subsection{Self-assembly of organosilanes: generalities}

The reaction of organosilane $\left(\mathrm{R}_{(4-n)}-\mathrm{Si}-\mathrm{X}_{n} ; n \in[1,2,3]\right)$ derivatives with hydroxylated surface has attracted attention for several years. $R$ is most often an alkyl chain, which may have 
different features, and $\mathrm{X}$ is an alkoxy or a chlorine. In the further study only organosilanes trifunctionalized $(n=3)$ will be used. They have the distinction of creating intermolecular bonds (fig. 24), which leads to the formation of an ordered molecular monolayer robust due to the crosslinking.

\subsubsection{Grafting mechanism}

The grafting process of organosilanes trifunctionalized takes place in four steps (fig. 24)[1].

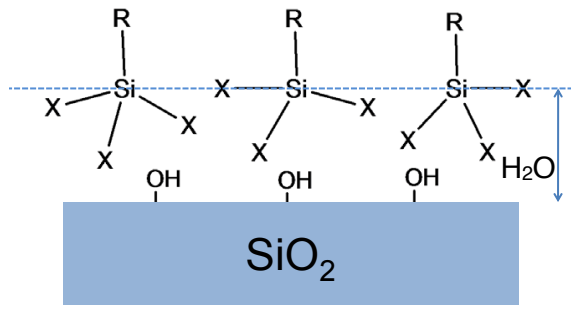

Step 1: Physisorption

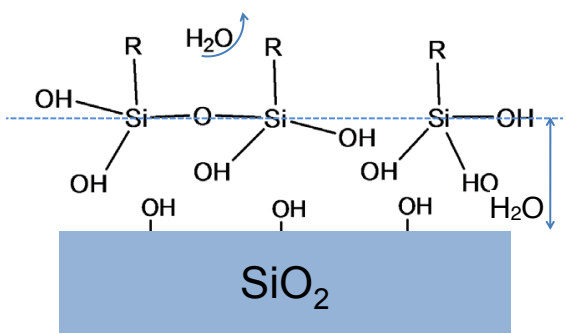

Step 3: In plane reticulation

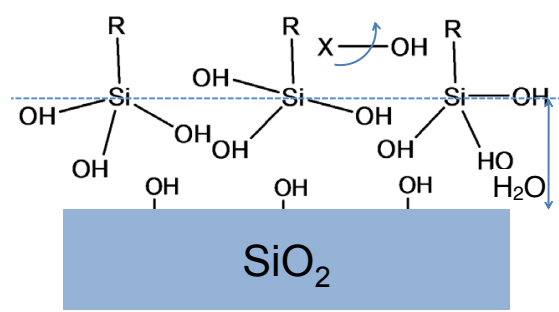

Step 2: Hydrolysis

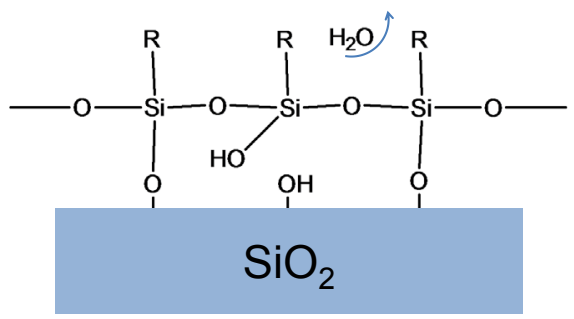

Step 4: Covalent grafting to the substrate

Figure 24. Different steps involved in the mechanism of SAM formation on a hydrated silica surface [1]

Step 1: Physisorption of molecules onto the adsorbed water monolayer(s) on the surface by the hydrophilic $\mathrm{Si}-\mathrm{R}_{3}$ moieties. Depending of the humidity rate there is one to three layer(s) of water on surface.

Step 2: Hydrolysis of the active-head group $\mathrm{Si}^{-} \mathrm{R}_{3}$ by reaction with water.

Step 3: In plane reticulation: the surface-active head groups of two molecules react, and the reaction entails the formation of intermolecular siloxane bond(Si-O-Si). This reticulation is essential to the formation of close-packed and well-ordered SAMs.

Step 4: During this step molecule will react vita the surface forming siloxane bond (Si-O-Si).

During the various steps, the molecules, isolated or not, can diffuse on top of the water layer present on the surface. Through the interactions between neighboring molecules (hydrophobic body and active-surface group) under certain conditions leading to growth by islands, this mobility allows the molecules to compact enough for the transition from step 2 to step 3 . Steps 3 and 4 are difficult to separate because they seem to occur almost simultaneously[8, 49]. 


\subsubsection{Molecular structure influence}

Each part of the molecule has an impact on the SAM growth[35]:

Surface-active head group: The kinetics of the monolayer formation is driven by the hydrolysis of the head group. Generally, the trifunctionalized organosilanes $\left(\mathrm{R}-\mathrm{Si}-\mathrm{X}_{3}\right)$ bear the same moieties $\mathrm{X}$. Three moieties are particularly used: chlorine, methoxy and ethoxy. The table 9 present a classification by decreasing reactivity with $\mathrm{SiO}_{2}$ as a function of the surface-active head group:

\begin{tabular}{cc}
\hline \hline silane moieties & name \\
\hline \hline $\mathrm{Si}-\mathrm{Cl}_{3}$ & trichlorosilane \\
$\mathrm{Si}-\left(\mathrm{OCH}_{3}\right)_{3}$ & trimethoxysilane \\
$\mathrm{Si}-\left(\mathrm{OCH}_{3}\right)_{2}\left(\mathrm{OC}_{2} \mathrm{H}_{5}\right)_{1}$ & dimethoxyethoxysilane \\
$\mathrm{Si}-\left(\mathrm{OCH}_{3}\right)_{1}\left(\mathrm{OC}_{2} \mathrm{H}_{5}\right)_{2}$ & methoxydiethoxysilane \\
$\mathrm{Si}-\left(\mathrm{OC}_{2} \mathrm{H}_{5}\right)_{3}$ & triethoxysilane \\
\hline
\end{tabular}

Table 9. Classification of silanes moieties by decreasing reactivity with $\mathrm{SiO}_{2}$ [40]

body group: The interchain van der Waals and electrostatic interactions are responsible of the final organization. The longer is the alkyl chain, the better is the organization. Note that beyond 18 carbons in the chain the SAMs are more disordered[1].

Surface group: This part of the molecule has few influence on the order. The use of large size groups can lead to steric hindrance.

\subsection{Self-assembly of organosilanes: methodology}

\subsubsection{Cleaning process}

Silicon substrates are cut from $\mathrm{Si}(100)$ wafers covered with native oxide. First, substrates are degreased in a sonicated chloroform bath (1) fig. 25), and then dried under a nitrogen flow(2) fig. 25). Substrates are then soaked into a piranha mixture $\left(\mathrm{H}_{2} \mathrm{SO}_{4}, \mathrm{H}_{2} \mathrm{O}_{2} 30 \%\right.$; (v/v) 7:3, highly exothermic reaction, caution!) for 30 minutes at $150{ }^{\circ} \mathrm{C}$ (3 fig. 25) in order to remove any organic impurities from the surface and to increase the amount of hydroxyl moieties $(\mathrm{OH})$ necessary for the grafting of silane heads. After that they are rinsed (4) fig. 25) with ultrapure deionized water $(18 \mathrm{M} \Omega . \mathrm{cm})$ and quickly immersed into a beaker of de-ionized water(5) fig. 25).

\subsubsection{Grafting process}

Still in this beaker, substrates are introduced into a glove-box filled with nitrogen at $40 \%$ relative humidity in which silanization is performed. Substrates are dried under a nitrogen flow (1) fig.26) and dipped into the silanization solution (2) fig. 26), consisting of a mixture of hexadecane, carbon tetrachloride and the trichlorosilane at $10^{-2} M$ total concentration. This solution was beforehand thermalized on a thermostated plate during $\sim 20$ minutes at $11^{\circ} \mathrm{C}$ to which it is kept during all the silanization time of 1.5 to 2 hours. Then, samples are rinsed in a sonicated chloroform bath (4) fig. 26) and dried under a nitrogen flux (5) fig. 26). 


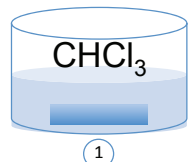

$\mathrm{t}=5 \mathrm{~min}$
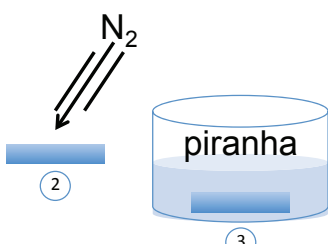

$\mathrm{t}=30 \mathrm{~min}$
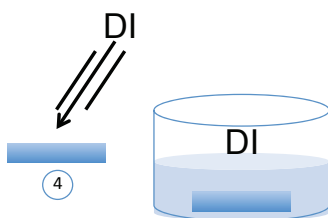

(5)

$\mathrm{t}=5 \mathrm{~min}$

Figure 25. Cleaning process of silicon substrates

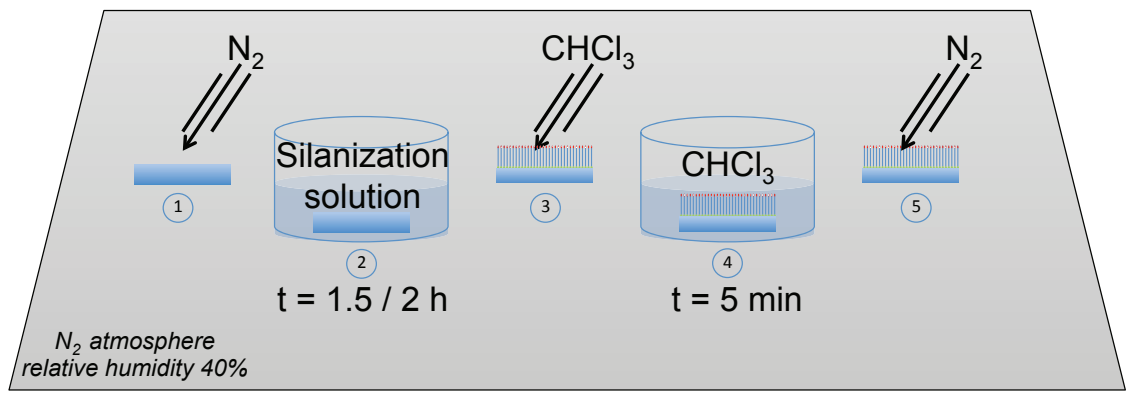

Figure 26. Silanization process

\subsection{Self-assembly of organosilanes: critical parameters}

In addition to the structure of the molecule and its density on the surface, the realization of a SAM from a solution involves other essential parameters to be controlled such as the nature of the solvent, the concentration, temperature and duration of the deposition which is conditioned by the kinetics reaction between the surface-active group and the surface.

\subsubsection{Substrate cleaning}

The silica surfaces are very sensitive to organic contaminants because of their high polarity. Simon Desbief, during his thesis at the IM2NP, tested different cleaning protocols (UV-ozone $+\mathrm{HF}, \mathrm{UV}$-ozone + HF + piranha, piranha ...) and their influence on the quality of an octadecyltrichlorosilane (OTS) layer. It appears that in the absence of a piranha treatment the layers obtained are very disorganized. The piranha removes most organic contaminants and makes the surface hydrophilic by promoting the formation of hydroxyl groups on the surface, i.e., attachment points of the molecules. On the other hand, results are not better when treatment is preceded by an attack of hydrofluoric acid and followed by a UV-ozonolysis.

\subsubsection{Temperature}

The grafting temperature is an important parameter. Contrary to what one might think, decreasing the grafting temperature favors the order of the layer. Brzoska et al. $[9,10]$ were the first to highlight this feature. They demonstrated the existence of a critical growth temperature $\left(T_{C}\right)$ above which the monolayer is disordered. This temperature increases linearly with the 
length of the alkyl chain of the molecule, $\mathrm{T}_{C}=K n$ where $n$ is the number of carbon forming the chain. Other studies have shown that the change of the type of growth does not occur at a temperature $\mathrm{T}_{C}$ but within a temperature range[ $\left.\mathrm{T}_{C 1} ; \mathrm{T}_{\mathrm{C} 2}\right]$. When the temperature of the solution is in this range, there is a competition between ordered and disordered growth modes. Sung et al. observed a change of the state of the layer depending on the temperature of the bath. They prepared a SAM at a temperature $\mathrm{T}<\mathrm{T}_{C}$, then soaked it into a bath at $\mathrm{T}>\mathrm{T}_{C}$ and then plunged it back into a bath at $\mathrm{T}<\mathrm{T}_{C}$. It appears that in this sequence the layer grows in patches and then is made of completely disordered structure islands. It also highlights a pre-organization of the layer before grafting to the surface.

\subsubsection{Solvent}

Solvent polarity plays a role on the reactivity of molecules. In fact, the speed of steps 2 et 4 (fig. 24) increases as a function of the polarity of the solvent used. Moreover, the solvent had to solubilize the molecules to prevent their aggregation in solution and at the same time it has to facilitate its transfer on the surface. During the synthesis of binary monolayer ${ }^{1}$, the molecule-solvent affinity allows to better control of the phase separation between the two molecules and thereby to facilitate the adsorption of a molecule with respect to the other.

\subsubsection{Hydrometry}

Water plays a key role during grafting. Indeed, studies have shown that the use of fully dehydrated substrates led to the formation of disordered layers, or conversely that the presence of water in the grafting solution activated the layer growth. Note that the presence of a big amount of water can cause polycondensation[11].

\subsubsection{Concentration}

The concentration of the grafting solution influences the kinetics of grafting. The more concentrated the solution is, the more molecules will interact with the hydroxyl groups of the surface. It should be noted that too high concentration may cause the formation and deposition of aggregates[11].

\subsection{Shape analysis}

It is thus interesting to statistically study the evolution in shape of such molecular islands according to various experimental parameters (time elapsed during the experiment, length of the molecule, temperature, etc.) in order to predict their further properties [36]. Images are obtained by microscopy with atomic force (AFM) and then analyzed. A model of identification card for a given island is then presented in Fig. 27. This card includes a number identifying the island, the location of its centroid, its perimeter and surface values, its feature values (CP: circularity parameter, dmax: maximal diameter, hmax: maximal height, $\bar{d}_{\text {max }}$ : average diameter), its representation in $2 \mathrm{D}$ and $3 \mathrm{D}$, and finally, the graphs of $P N$ (point numbers from the boundary) and $V H$ (average height variations). Then, a set of identification cards can be gathered in order to get statistical data.

\footnotetext{
${ }^{1}$ In this case there are 2 kinds of molecules in the silanization solution.
} 


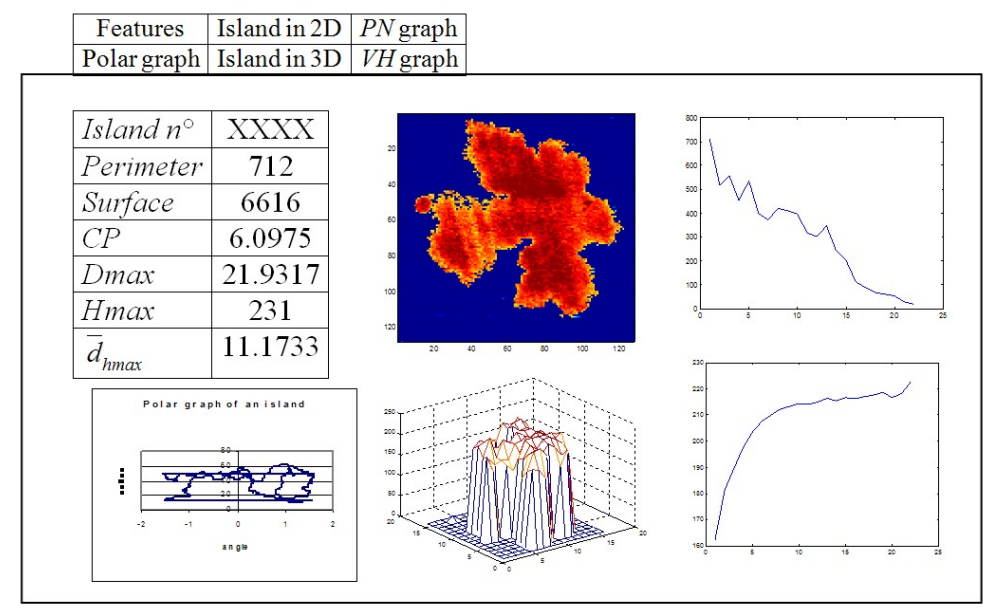

Figure 27. Identification card of an island

\section{Conclusion}

In this chapter, a similarity parameter has been presented in order to compare shapes. Other parameters have been considered to determine shape features and their efficiency has been tested in real situations. Shape classification is achieved in an automatic way, that enables the processing of a huge amount of data, as well as statistical studies. The process has been illustrated in two different application contexts. For nuclear fuel particles, the estimation of the sphericity degree gives a mean of detection of what step of the fabrication is defective or deviating. This allows to characterize a sample of particles in order to determine if the batch they belong should be rejected or not. For molecular islands, the parameters define the identification card of each bounded surface. This leads to a statistical study in shape according to the time elapsed during the experiment.

\section{Author details}

F. Robert-Inacio and G. Delafosse

Institut Supérieur d'Electronique et du Numérique, IM2NP

CNRS, IM2NP (UMR 7334)

\section{Patrone}

Institut Supérieur d'Electronique et du Numérique, IM2NP

CNRS, IM2NP (UMR 7334)

Aix-Marseille Université, IM2NP, Maison des Technologies, Place Georges Pompidou, F-83000 Toulon, France

\section{References}

[1] Aswal, D. K., Lenfant, S., Guerin, D., Yakhmi, J. V. \& Vuillaume, D. [2006]. Self assembled monolayers on silicon for molecular electronics, Analytica chimica acta 568(1-2): 84-108. 
[2] Avis, D., Bremner, D. \& Seidel, R. [1997]. How good are convex hull algorithms, Computational Geometry: Theory and Applications 7: 265-302.

[3] Baptiste, A., Gibaud, A., Bardeau, J. F., Wen, K., Maoz, R., Sagiv, J. \& Ocko, B. M. [2002]. X-ray, Micro-Raman, and infrared spectroscopy structural characterization of Self-Assembled multilayer silane films with variable numbers of stacked layers, Langmuir 18(10): 3916-3922.

[4] Barber, C. B., Dobkin, D. \& Huhdanpaa, H. [1996]. The quickhull algorithm for convex hulls, ACM Trans. Math. Softw. 22(4): 469-483.

[5] Blaschke, W. [1923]. Vorlesungen über Differentialgeometrie. II: Affine Differentialgeometrie, Springer, Berlin.

[6] Blodgett, K. B. [1935]. Films built by depositing successive monomolecular layers on a solid surface., J. Amer. chem. Soc. 57: 1007-1022.

[7] Blodgett, K. B. \& Langmuir, I. [1937]. I.: Built-up films of barium stearate and their optical properties., Physical Rev. 51: 964-982.

[8] Brochier Salon, M. \& Belgacem, M. N. [2010]. Competition between hydrolysis and condensation reactions of trialkoxysilanes, as a function of the amount of water and the nature of the organic group, Colloids and Surfaces A: Physicochemical and Engineering Aspects 366(1-3): 147-154.

URL: $h t t p: / / w w w . s c i e n c e d i r e c t . c o m / s c i e n c e / a r t i c l e / p i i / S 0927775710003389$

[9] Brzoska, J. B., Azouz, I. B. \& Rondelez, F. [1994]. Silanization of solid substrates: A step toward reproducibility, Langmuir 10: 4367-4373.

[10] Brzoska, J. B., Shahidzadeh, N. \& Rondelez, F. [1992]. Evidence of a transition temperature for the optimum deposition of grafted monolayer coatings, Nature 360: 719-721.

[11] Bunker, B. C., Carpick, R. W., Assink, R. A., Thomas, M. L., Hankins, M. G., Voigt, J. A., Sipola, D., de Boer, M. P. \& Gulley, G. L. [2000]. The impact of solution agglomeration on the deposition of Self-Assembled monolayers, Langmuir 16(20): 7742-7751.

URL: $h t t p: / / d x$.doi.org/10.1021/la000502q

[12] Charollais, F., Duhart, A. Felines, P., Guillermier, P. \& C., P. [2004]. Influence of thermal treatment conditions on microstructure and composition of htr fuel kernel, Ceramics Transactions of Am Ceram Soc 168: 109-118.

[13] Charollais, F., Fonquernie, S., Perrais, C., Perez, M., Cellier, F. \& Harbonnier, G. [2004]. Cea and areva $\mathrm{r}$ and $\mathrm{d}$ on htr fuel fabrication and presentation of the gaia experimental manufacturing line, Proceedings of HTR2004, Beijing, China.

[14] Charollais, F., Perez, M., Fonquernie, S., Ablitzer, C., Duhart, A., Perrais, C., Dugne, O., Guillermier, P. \& Harbonnier, G. [2004]. Cea and areva fuel particles manufacturing and characterization $r$ \& d program, Proceedings of Atalante2004, Nimes, France.

[15] Choi, K. [2000]. Controlled deposition of carbon nanotubes on a patterned substrate, Surface Science 462(1-3): 195-202.

[16] Danielsson, P. [1980]. Euclidean distance mapping, CVGIP 14: 227-248.

[17] Duchene, S., Davidovits, N. \& Millasseau, F. [n.d.]. Brevet Français numéro 960.1319.

[18] Gauthier, J., Lecomte, M., Brinkmann, G. \& B., B. C. [2004]. Antares: the htr/vhtr project at framatome anp, Proceedings of 2nd int meeting on HTR Techn 2004.

[19] Grünbaum, B. [1963]. Measures of symmetry for convex sets, Proc. Symp. Pure Math., Vol. 7, pp. 233-270. 
[20] H.Lee, Kepley, L., Hong, H. \& Mallouk, T. [1987]. Inorganic analogues of Langmuir-Blodgett films: Adsorption of ordered zirconium 1,10-decanebisphosphonate multilayers on silicon., J Am. Chem. Soc. 110: 618-620.

[21] Ho, C. \& Chen, L. [1995]. A fast ellipse/circle detector using geometric symmetry, Pattern Recognition 28(1): 117-124.

[22] Inacker, O., Kuhn, H., Mobiusand, D. \& Debuch, G. [1976]. Manipulation in molecular dimensions., Z. physik. Chem. Neue Folge 51: 337-360.

[23] Kazhdan, M., Chazelle, B., Dobkin, D., Finkelstein, A. \& Funkhouser, T. [2002]. A reflective symmetry descriptor, Proc. European Conference on Computer Vision (ECCV).

[24] Kazhdan, M., Funkhouser, T. \& Rusinkiewicz, S. [2004]. Symmetry descriptors and 3D shape matching, R. Scopigno and D. Zorin editors.

[25] Kim, H. I., Graupe, M., Oloba, O., Koini, T., Imaduddin, S., Lee, T. R. \& Perry, S. S. [1999]. Molecularly specific studies of the frictional properties of monolayer film : a systematic comparaison of $\mathrm{cf}_{3},\left(\mathrm{ch}_{3}\right)_{2} \mathrm{ch}$, and $\mathrm{ch}_{3}$ terminated films., Langmuir 15(9): 3179-3185.

[26] Labouré, M., Jourlin, M., Fillère, I., Becker, J. \& Frénéa, F. [1996]. Isoperimetric inequalities and shape parameters, Acta Stereologica 15/1: 65-70.

[27] Languille, A. [2002]. High temperature reactor fuel technology programme in europe, Proceedings of HTR-TN 2002, Petten, Holland.

[28] Mann, B. \& Kuhn, H. [1971]. Tunneling through fatty acid salt monolayers, Journal of Applied Physics 42: 4398.

[29] Maoz, R., Matlis, S., DiMasi, E., Ocko, B. M. \& Sagiv, J. [1996]. Self-replicating amphiphilic monolayers, Nature 384: 150-153.

URL: http://www.nature.com/nature/journal/v384/n6605/pdf/384150a0.pdf

[30] Maoz, R., Sagiv, J., Degenhardt, D., Miihwald, H. \& Quint, P. [1995]. Hydrogen-bonded multilayers of self-assembling silanes: structure elucidation by combined fourier transform infra-red spectroscopy and x-ray scattering techniques, Supramolecular Science 2(1): 9-24.

[31] Marola, G. [1989]. On the detection of the axis of symmetry or almost symmetric planar images, IEEE PAMI 11: 104-107.

[32] Netzer, L., Iscovici, R. \& Sagiv, J. [1983]. Adsorbed monolayers versus langmuir-blodgett monolayers- why and how ? 1: From monolayer to multilayer by adsorption., Thin Solid Films 99: 235-241.

[33] Phelip, M. [2003]. First results from htr-f\&f1 projects : Htr fuel technology, Proceedings of International Congress on Advanced nuclear Power Plants ICAPP'03, Cordoba, Spain.

[34] Piner, R. D., Zhu, J., Xu, F., Hong, S. \& Mirkin, C. A. [1999]. "Dip-Pen" nanolithography, Science 283(5402): $661-663$.

[35] Rauscher, H. [2001]. The interaction of silanes with silicon single crystal surfaces: microscopic processes and structures, Surface Science Reports 42(6-8): 207-328.

[36] Robert-Inacio, F. [2008]. Shape studies in 2d and 3d on molecular islands of carbon chains self-assembled on silicon in nanoelectronics, European Physical Journal: Applied Physics 41/1: 53-68.

[37] Robert-Inacio, F., Boschet, C., Charollais, F. \& Cellier, F. [2006]. Polar studies of the sphericity degree of $\mathrm{v} / \mathrm{htr}$ nuclear fuel particles, Materials Characterization 56(4-5): 266-273. 
[38] Rondelez, F. \& Bezou, P. [1999]. Des surfaces antibactériennes et autostériles, L'actualité chimique 10: 4-8.

[39] Schreiber, F. [2000]. Structure and growth of self-assembling monolayers, Progress in Surface Science 65(5-8): 151-257.

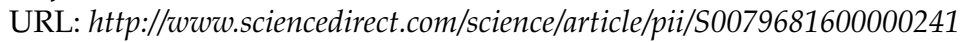

[40] Simon, A. [2002]. Intérêt de la microscopie de force atomique sur la bio fonctionnalisation de matériaux : caractérisation du greffage et de l'adhésion cellulaire., $\mathrm{PhD}$ thesis, Université Bordeaux I.

[41] Sugimura, H. \& Nakagiri, N. [1997]. Nanoscopic surface architecture based on scanning probe electrochemistry and molecular Self-Assembly, J. Am. Chem. Soc. 119(39): 9226-9229.

[42] Sun, C. [1995]. Symmetry detection using gradient information, Pattern Recognition Letters 16: 987-996.

[43] Sun, C. \& Si, D. [1999]. Fast reflectional symmetry detection using orientation histograms, Real-time Imaging 5: 63-74.

[44] Süss, W. [1950]. Ueber eibereiche mit mittelpunkt, Math-Phys Semesterber 1: 273-287.

[45] Ulman, A. [1991]. An Introduction to ULTRATHIN ORGANIC FILMS From Langmuir-Blodgett to Self-Assembly, ACADEMIC PRESS, Inc, London.

[46] Vuillaume, D., Boulas, C., Collet, J., Davidovits, J. V. \& Rondelez, F. [1996]. Organic insulating films of nanometer thicknesses, Applied Physics Letters 69: 1646.

[47] Wang, H. \& Suter, D. [2003]. Using symmetry in robust model fitting, Pattern Recognition Letters 24: 2953-2966.

[48] Wolter, J., Woo, T. \& Volz, R. [1985]. Optimal algorithms for symmetry detection in two and three dimensions, The Visual Computer 1: 37-48.

[49] Yang, Y., Bittner, A. M., Baldelli, S. \& Kern, K. [2008]. Study of self-assembled triethoxysilane thin films made by casting neat reagents in ambient atmosphere, Thin Solid Films 516(12): 3948-3956.

URL: http://www.sciencedirect.com/science/article/pii/S0040609007013685 